\title{
Levantamento de imaturos de libélulas (INSECTA: ODONATA) em tanques escavados de piscicultura na mesorregião de Alfenas-MG
}

Survey of dragonfly immature (INSECTA: ODONATA) in excavated tanks of pisciculture in the mesoregion of Alfenas-MG

Relevamiento de libélulas inmaduras (INSECTA: ODONATA) en estanques piscícolas excavados en la mesorregión de Alfenas-MG

\section{Resumo}

Imaturos de libélula são encontrados em uma vasta quantidade de ambientes aquáticos de água doce, dentre eles, os tanques escavados para piscicultura. Como são predadores durante a fase aquática, acabam por causar grandes prejuízos nas fases iniciais de produção da aquicultura de água doce. No entanto, os estudos de quais são as libélulas mais comuns em tanques de piscicultura ainda são escassos no Brasil, sendo que este é primeiro passo para se pensar num controle eficaz. Por esse motivo, coletamos imaturos de libélula (Odonata) com rede "D" e macrófitas entre outubro de 218 e março de 2019. As variáveis físico-químicas foram medidas com kits de qualidade da água, sendo que se mantiveram dentro dos parâmetros para piscicultura definidos pelo CONAMA, com exceção da temperatura. Dos 692 imaturos coletados, encontramos 12 gêneros distribuídos nas duas subordens de Odonata, sendo Miathyria Kirby, 1889 e Erythemis Hagen, 1861 os mais abundantes. Após análise de correspondência e análise de componentes principais, comprovamos que os gêneros mais raros têm menor correlação com a abundância, reforçando o valor atribuído a abundância relativa dos imaturos.

Palavras-chave: Erythemis; Miathyria; Libellulidae; Odonata; Larvicultura; Piscicultura; Aquicultura.

\begin{abstract}
Dragonfly immatures are found in a vast number of freshwater aquatic environments, including excavated tanks of pisciculture. As they are predators during the aquatic phase, they end up causing great damage in the initial stages of production of freshwater aquaculture. However, studies on which dragonflies are most common in fish ponds are still scarce in Brazil, and this is the first step to think about an effective control. For this reason, we collected immature dragonfly (Odonata) with net "D" and macrophytes between October 218 and March 2019. The physicochemical variables were measured with water quality kits, and they remained within the parameters for fish farming defined by CONAMA, with the exception of temperature. Of the 692 immatures collected, we found 12 genera distributed in the two suborders of Odonata, with Miathyria Kirby, 1889 and Erythemis Hagen, 1861 being the most abundant. After
\end{abstract}


correspondence analysis and principal component analysis, we proved that rarer genera have a lower correlation with abundance, reinforcing the value attributed to the relative abundance of immatures.

Keywords: Erythemis; Miathyria; Libellulidae; Odonata; Larviculture; Fisheries; Aquiculture.

\begin{abstract}
Resumen
Las libélulas inmaduras se encuentran en una gran cantidad de ambientes acuáticos de agua dulce, incluidos estanques de peces excavados. Al ser depredadores durante la fase acuática, terminan causando grandes daños en las etapas iniciales de producción de la acuicultura de agua dulce. Sin embargo, los estudios sobre qué libélulas son más comunes en los estanques de peces aún son escasos en Brasil, y este es el primer paso para pensar en un control efectivo. Por este motivo, recolectamos libélula inmadura (Odonata) con red "D" y macrófitas entre el 218 de octubre y marzo de 2019. Las variables fisicoquímicas se midieron con kits de calidad de agua, y se mantuvieron dentro de los parámetros para piscicultura definidos por CONAMA, con el excepción de la temperatura. De los 692 inmaduros recolectados, encontramos 12 géneros distribuidos en los dos subórdenes de Odonata, siendo Miathyria Kirby, 1889 y Erythemis Hagen, 1861 los más abundantes. Después del análisis de correspondencia y el análisis de componentes principales, demostramos que los géneros más raros tienen una menor correlación con la abundancia, reforzando el valor atribuido a la abundancia relativa de inmaduros.
\end{abstract}

Palabras clave: Erythemis; Miathyria; Libellulidae; Odonata; Larvicultura; Piscicultura; Acuicultura.

\title{
1. Introdução
}

A aquicultura tem apresentado elevado crescimento condicionado a uma maior intensificação e modernização dos sistemas de produção, proporcionando maiores índices de produtividade, sendo o setor de produção de alimentos que mais cresce no mundo. Este aumento acentuado também está relacionado à escassez do pescado natural e preocupação atual da população com a segurança alimentar (Kirchner et al., 2016; Mello et al., 2017; Fao et al., 2018; Lui et al., 2018; Siqueira, 2018).

No Brasil, a área de maior sucesso dentro da aquicultura é a piscicultura e isso se deve às características favoráveis como clima, território disponível, entre outras que propiciam a produção de diversas espécies nativas e exóticas. A tilápia (Oreochromis niloticus (LINNAEUS, 1758)), uma espécie africana, se adaptou bem às condições do país e hoje é a espécie mais produzida. Dentre os nativos, o tambaqui (Colossoma macropomum (CUVIER, 1818)) e seus híbridos se destacam na produção (tambacu, cruzamento da fêmea de tambaqui com macho de pacu (Schulter \& Vieira Filho, 2017).

A piscicultura ornamental também está presente no Brasil, que é o oitavo mercado de exportação do mundo. No entanto, o levantamento preciso dessa cadeia produtiva ainda é baixo, sendo executado gradativamente em alguns estados por meio de publicações de artigos científicos ou dados não oficiais (De Faria et al., 2019).

A piscicultura possui diferentes sistemas de produção, extensivo, intensivo e superintensivo, o sistema extensivo se utiliza de ambientes próximos aos naturais e com pouca ou nenhuma suplementação alimentar, o sistema intensivo utiliza corpos d'água escavados para esta finalidade e com suplementação alimentar de quase todo alimento consumido pelos peixes, o sistema superintensivo se baseia em tanques de alvenaria, geralmente em estufas e com suplementação de todo o alimento dos peixes (Americo et al., 2013; Sebrae, 2015; Siqueira, 2018).

O sistema intensivo de cultivo ainda acaba sendo o mais utilizado pois tem um custo bem menor que as estufas utilizadas no sistema superintensivo. Entretanto, como os tanques do sistema intensivo são escavados e estão a céu aberto, estão sujeitos a colonização por agentes externos, no geral, insetos que voam na fase adulta e colonizam ambientes diferentes (Kubitza, 2011).

A reprodução comercial de tilápias precisa ter alevinos de idade semelhante e em quantidade para atender a demanda, outro ponto é que essa espécie atinge a maturidade sexual num tamanho menor nas fêmeas que nos machos, por isso, durante a fase de alevinagem, há também a reversão sexual para que os alevinos sejam em sua vasta maioria, machos, há diversas formas de se fazer essa reversão, mas a menos impactante, é, sem dúvidas, a reversão por controle de temperatura da água na fase em que as gônadas estão sendo formadas (Tachibana et al., 2004; Igarashi, 2018; Tachibana et al., 2018). 
Os adultos ficam em tanques onde fazem os ninhos e as fêmeas incubam os ovos na boca, nesta fase, os ovos fertilizados são retirados da boca das fêmeas e transferidos para incubadoras artificiais até a eclosão (SENAR nº197, 2017). As larvas eclodidas permanecem no laboratório em bandejas até que o saco vitelínico seja consumido para completar a organogênese, culminando com a abertura da boca, após esse processo, os peixes são considerados pós-larvas e já podem receber alimentação externa (SENAR n¹97, 2017; SENAR, n¹98, 2017). Geralmente às pós-larvas são estocadas nos tanques onde já podem receber ração e nessa fase, dependendo do seu tamanho e sensibilidade, os peixes ficam suscetíveis aos predadores (Kubitza, 2011).

A reprodução comercial de peixes nativos depende, na sua maioria, de um laboratório capaz de fazer a indução hormonal a maturação final das gônadas para obtenção dos gametas que são então colocados juntos para que haja a fecundação (Andrade \& Yasui, 2003). Após a fecundação vai haver a formação da membrana coriônica via hidratação do ovo. Os ovos são então levados para incubadoras com circulação de água e adaptadas a espécie que se está reproduzindo, após algumas horas, uma larva que ainda não completou a formação dos órgãos e ainda tem uma reserva (saco vitelínico), eclode para o meio externo, essa fase é marcada pelos movimentos de natação no sentido vertical dentro da incubadora (Zaniboni Filho \& Weingartner, 2007; Da Silva et al., 2009).

Essa larva permanece na incubadora até que tenha a formação dos olhos, formação de vários órgãos e abertura da boca, quando então poderá receber alimentação externa, assim que a boca se abre, as larvas irão formar a bexiga natatória e passam a nadar tanto na vertical quanto na horizontal dentro da incubadora (Da Silva et al., 2009). Como a boca já está aberta e as reservas do ovo foram consumidas, estas larvas são, então, levadas para tanques onde se alimentarão de plâncton e ração de tamanho adequado para a espécie (Kubitza, 2004).

A larvicultura é o processo em que as pós-larvas serão estocadas em tanques, alimentadas com ração e plâncton e irão completar seu desenvolvimento ficando mais parecidos com os peixes adultos e recebendo o nome de alevinos, por isso que esse processo recebe o nome de larvicultura e alevinagem (Meurer et al., 2003; SENAR nº198, 2017). Durante a larvicultura e alevinagem, como os animais estão em tanques, vários predadores podem afetar esse processo, desde peixes invasores de lagoas próximas, girinos, pássaros, bem como invertebrados aquáticos, como Copepoda e insetos aquáticos (Kubitza, 2011). Dentre esses insetos, os imaturos de libélulas (Odonata), os Hemiptera: Notonectidae (patinadores) e Belostomatidae (percevejo aquático gigante) podem viver tanto na região bentônica, coluna d'água e até mesmo na superfície e podem infestar os tanques escavados quando não há um preparo adequado, que na maioria das vezes é feito com organofosforados (Lopes et al., 2007; Queiroz, 2017). As larvas de Odonata podem ser consideradas o predador de peixes mais importante nos estágios iniciais de vida dos peixes (Kubitza, 2003; 2011).

Existem três subordens de Odonata: Anisozygoptera (restrita à região asiática), Anisoptera e Zygoptera. Os adultos da subordem Anisoptera podem ter bases diferentes das asas dianteiras e traseiras, pousando com elas abertas e os adultos da subordem Zygoptera podem ter as bases das asas semelhantes, mas pousam com as asas fechadas nas costas (Souza et al., 2007). Quanto aos imaturos, eles se desenvolvem em ambientes de água doce, os zigópteros têm corpo delgado, com três lamelas caudais (brânquias) no final do abdome, frequentemente utilizadas para nadar, enquanto os anisópteros têm corpo robusto, não possuem os filamentos caudais e respiram pelas brânquias retais da traqueia, a entrada e a saída da água pelo ânus servem para a respiração e a propulsão rápida do corpo quando o abdome é contraído (Souza et al., 2007).

Esses insetos são hemimetábolos e predadores tanto na fase adulta quanto na fase imatura, segundo Paulson \& Schorr (2021) 6.317 espécies são conhecidas no mundo, das quais 749 ocorrem no Brasil (Olaya, 2019). Possuem dependência direta da água quando imaturos e dependência indireta da água quando adultos, por estarem sempre associados a ambientes límnicos e também para reprodução (Queiroz, 2017). As espécies de Odonata variam no número de estágios imaturos até a emergência, podendo passar por períodos entre um mês, a mais de um ano até atingirem a fase adulta (Borror et al., 1989; Costa et al., 
2012; Ramirez et al., 2010; Souza et al., 2007; Trueman \& Rowe, 2009). Durante o tempo de desenvolvimento desses insetos até a fase adulta, eles podem causar danos significativos à larvicultura e alevinagem de peixes, afetando parte ou toda produção. (Tave et al., 1990).

Devido aos problemas mencionados causados pela predação de Odonata na piscicultura, torna-se importante conhecer os gêneros e espécies desses insetos aquáticos mais prevalentes nesses ambientes a fim de mitigar soluções sustentáveis para o controle, porém, os estudos ainda são escassos e sua maioria trata de uma mesma espécie, Pantala flavescens (FABRICIUS, 1798) (Santos et al., 1988; De Marco et al., 1999; Fonseca et al., 2004), sendo que essa espécie não foi a mais prevalente neste estudo, indicando a necessidade de ampliar os conhecimentos sobre quais imaturos de Odonata estão presentes em cada ecoregião do Brasil.

Portanto, o objetivo deste trabalho foi realizar um levantamento dos gêneros Odonata em tanques de piscicultura no sul de Minas Gerais, Brasil, na mesorregião de Alfenas-MG.

\section{Metodologia}

O trabalho foi realizado no campus da Universidade José do Rosário Vellano em Alfenas ( $21^{\circ} 27 ' 25,1$ "S $45^{\circ}$ 56'38,9" W), Estado de Minas Gerais, Brasil, entre outubro de 2018 e março de 2019 em cinco tanques escavados de piscicultura (Figura 1), com vegetação nativa, secundária e em regeneração nas proximidades e mata- interior (Mata Atlântica Semidescidual) (Veloso et al., 1991).

Os tanques 1 e 2 continham adultos de tilápia (Oreochromis niloticus (Linnaeus, 1758)) e curimbatá (Prochilodus lineatus (Valenciennes, 1837)) (curimba) e também estavam recobertos por macrófitas (Salvinia auriculata Aubl.). Os tanques 3, 4 e 5 destinavam-se a pós-larvas e alevinos de tilápia com presença considerável de macrófitas (S. auriculata, sendo que o tanque 3 havia a presença de Thypha sp. embora os insetos tenham sido coletados apenas S. auriculata).

Às coletas foram realizadas com rede "D" malha de $300 \mu \mathrm{m}$ para a coleta das larvas de Odonata com tempo padronizado de três minutos (Brandão et al., 2018). Em cada mês, havia uma coleta em um único dia quando foram feitas quatro amostragens em cada tanque escavado, sendo duas em macrófitas (S. auriculata) e duas no substrato (uma no substrato das margens outro na região bentônica).

Às amostras foram colocadas em recipientes de 2,5 L, fixadas em álcool 70\%, triadas em estereomicroscópio e identificadas por gênero de acordo com o manual de identificação de Thorp \& Covich (2018) Libelluloidea (Neiss et al., 2018); Aeshnoidea (Carvalho \& Pessacq, 2018); Coenagrioinoidea (Lozano et al., 2018). Nos dias das coletas, foram medidos os parâmetros físico-químicos da água como amônia tóxica, pH, oxigênio dissolvido e nitrito com os kits da (LABCON PET®) e os dados de temperatura com um termômetro digital (Instrusul). Os dados meteorológicos relativos aos dias de coletas da estação meteorológica da região de Alfenas-MG foram adquiridos na estação meteorológica da Cooperativa Regional dos Cafeicultores de Guaxupé em Alfenas-MG (Cooxupé, 2020).

Os dados bióticos e abióticos foram padronizados e analisados por Análise Correspondência Simples para correlacionar os gêneros com os tanques de piscicultura e para correlação das variáveis físico-químicas com os tanques, foi utilizada a Análise de Componentes Principais (ACP). Para todas as análises e tabulação dos dados foram utilizados o PAST Paleontological Statistics Software Package (Hammer, 2018) e o Office Software, Microsoft Excel.

Vale ressaltar que as datas de coleta foram escolhidas de acordo com o período climático considerado ideal para a reprodução dos peixes e coincidente com a dinâmica reprodutiva de Odonata (de Marco \& Resende, 2002; Silva et al., 2016; Mendonça et al., 2018). 
Figura 1. Tanques escavados de piscicultura da Universidade José do Rosário Vellano onde houveram às coletas dos gêneros de Odonata entre os meses de outubro/2018 a março/2019. 1a: Vista da entrada 1b: Vista lateral.
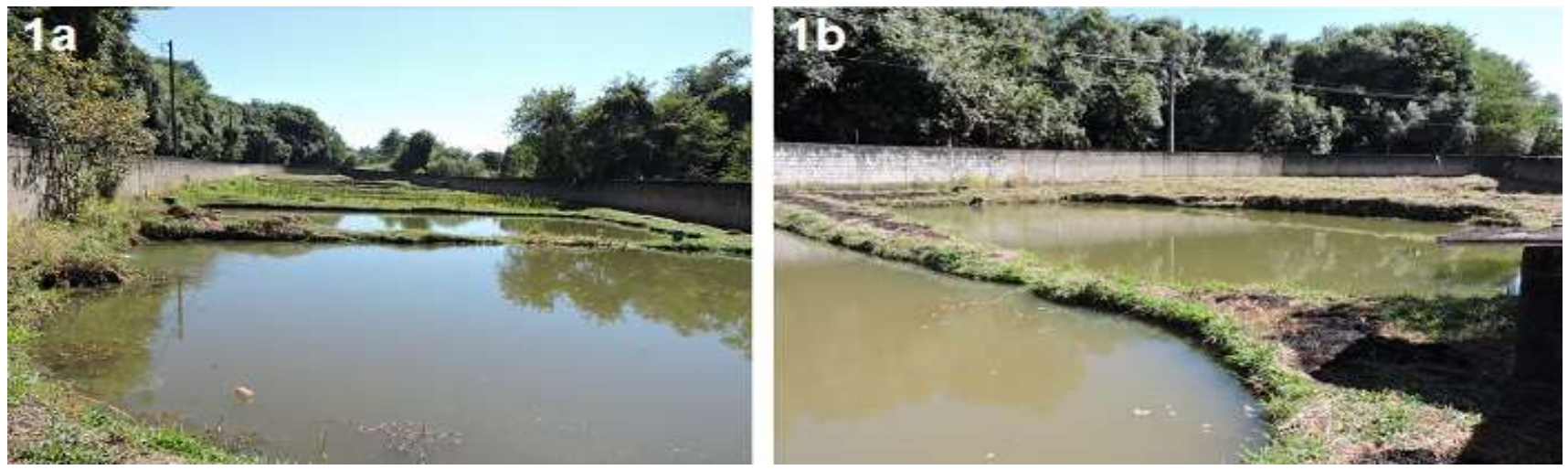

Fonte: Autores.

\subsection{Caracterização de tanques de piscicultura}

Os tanques de piscicultura onde ocorreram as coletas eram heterogêneos em tamanho, sendo o tanque 1 com 24 m por 13,90 por $1,50 \mathrm{~m}$ de profundidade; tanque $2: 24,25 \mathrm{~m}$ por $14,19 \mathrm{~m}$ por $1,50 \mathrm{~m}$ de profundidade; tanque $3: 24,13 \mathrm{~m}$ por $12,22 \mathrm{~m}$ por $1,50 \mathrm{~m}$ de profundidade; tanque $4: 20,45 \mathrm{~m}$ por $12,14 \mathrm{~m}$ por $1,50 \mathrm{~m}$ de profundidade e tanque $5: 17,14 \mathrm{~m}$ por $14,22 \mathrm{~m}$ por $1,50 \mathrm{~m}$ de profundidade.

Entre os meses da coleta, de acordo com o tamanho $\left(\mathrm{m}^{2}\right)$ dos tanques, foi calculada a proporção aproximada de macrófitas da espécie S. auriculata. Primeiramente, os dados foram obtidos pelo GOOGLE MAPS. Em seguida, foram realizadas medições das áreas dos grupos dessas macrófitas nos tanques, utilizando uma fita de fibra de vidro de $20 \mathrm{~m}$ (EDA) e, em seguida foi calculado o percentual. Tanque 1: come 4,3\% de cobertura, tanque 2 com $6,5 \%$, tanque 3 com $15 \%$, tanque 4 com $8,7 \%$ e tanque 5 com $9,35 \%$.

\section{Resultados e Discussão}

Foram encontrados doze gêneros de Odonata (Tabela 1), com dominância numérica de gêneros de Libellulidae, sendo o mais frequente foi Miathyria Kirby, 1889, com 34,41\%, seguido por Erythemis Hagen, 1861 com 20,4\%. O Predomínio de Libellulidae nos tanques de piscicultura corrobora com os trabalhos que foram desenvolvidos na área (Fonseca et al., 2004; Santos et al., 1998; De Marco et al., 1999).

Em relação a diferença numérica entre indivíduos nos tanques, foi possível observar que onde haviam peixes adultos (tanques 1 e 2) o número total de larvas de Odonata foi muito menor que nos tanques 3, 4 e 5 onde eram criadas larvas de tilápia, pós-larvas e alevinos. Este baixo número pode estar relacionado ao consumo de Odonata causado por peixes adultos (apesar das duas espécies não serem eficientes predadores), a tilápia tem hábito de filtro onívoro/ fitoplanctônico, alimenta-se próximo à superfície onde pode se nutrir das larvas dos insetos presentes nas raízes das macrófitas, enquanto a curimba, que é onívora/ilófaga, pode se alimentar na região bentônica (substrato) e consome as larvas de Odonata dessa região (Ostrensky \& Boeger, 1998; Sandoval Junior, 2019).

Outro fator que também pode estar relacionado à essa menor frequência de Odonata nos tanques 1 e 2 é a baixa biomassa de macrófitas. Em ecossistemas aquáticos, a presença de macrófitas contribui para um aumento considerável da área disponível para colonização por invertebrados (Pinder, 1995). 
Tabela 1. Gêneros, número total de larvas e percentual de Odonata (Insecta) encontradas em cinco tanques de piscicultura do sul de Minas Gerais entre os meses de outubro/2018 e março/2019.

\begin{tabular}{|c|c|c|c|c|c|c|c|}
\hline & $\begin{array}{c}\text { Tanque } \\
1\end{array}$ & Tanque 2 & Tanque 3 & Tanque 4 & Tanque 5 & Total & $\%$ \\
\hline \multicolumn{8}{|l|}{ AESHNIDAE } \\
\hline Anax Leach, 1815 & - & - & - & 2 & - & 2 & 0,28 \\
\hline \multicolumn{8}{|l|}{ COENAGRIONIDAE } \\
\hline Achantagrion Selys, 1876 & - & - & 9 & 6 & 6 & 21 & 3,03 \\
\hline Ischnura Charpentier, 1840 & - & - & 5 & 6 & - & 11 & 1,58 \\
\hline \multicolumn{8}{|l|}{ LIBELLULIDAE } \\
\hline Brachymesia Kirby, 1889 & - & - & 13 & 17 & 9 & 39 & 5,63 \\
\hline Erythemis Hagen, 1861 & 15 & 13 & 39 & 34 & 44 & 145 & 20,95 \\
\hline Erythrodiplax Brauer, 1868 & 2 & 1 & 13 & 10 & 3 & 29 & 4,19 \\
\hline Miathyria Kirby, 1889 & 19 & 16 & 66 & 60 & 79 & 240 & 34,68 \\
\hline Micrathyria Kirby, 1889 & 5 & 2 & 11 & 1 & 3 & 22 & 3,17 \\
\hline Orthemis Hagen, 1861 & 9 & 5 & 16 & 6 & 9 & 45 & 6,5 \\
\hline Pantala Hagen, 1861 & 9 & 12 & 24 & 21 & 17 & 83 & 11,99 \\
\hline Perithemis Hagen, 1861 & - & 1 & 8 & 16 & 10 & 35 & 5 \\
\hline Tauriphila Kirby, 1889 & - & - & 8 & 8 & 4 & 20 & 2,89 \\
\hline
\end{tabular}

Fonte: Dados da pesquisa.

Em relação aos microhabitats onde ocorreram às amostragens (Tabela 2), os gêneros Miathyria, Micrathyria, Perithemis, Brachymesia, Tauriphila, Anax, Achantagrion e Ischnura foram predominantes nas macrófitas e no substrato das margens e fundo Erythemis, Pantala, Orthemis e Erythrodiplax. Miathyria foi numericamente maior nas macrófitas, mas também foi considerada relevante no substrato.

A presença de Miathyria é comum em tanques de piscicultura, segundo Santos et al. (1988), que, em levantamento de Odonata em tanques de produção em Pirassununga - SP, observaram maior abundância de Pantala, porém com presença considerável de Miathyria, sendo que todos os imaturos em seu trabalho, foram coletados no substrato de fundo, pois as margens eram de concreto, sem macrófitas nas margens e nem mesmo flutuantes, impossibilitando a postura endofítica.

Fonseca et al. (2004) coletando Odonata em duas pisciculturas no estado de Minas Gerais, observou também que entre o total de imaturos amostradas em três meses de coletas, Pantala flavescens representou 44\%, seguida por Brachymesia furcata Hagen, 1861 e Perithemis mooma Kirby, 1889, porém Miathyria, o gênero mais comum desse trabalho, não foi encontrado em suas formas imaturas em sua pesquisa. Segundo o autor, indivíduos do gênero podem ter migrado para outros ambientes aquáticos próximos para postura.

O gênero Erythemis, foi encontrado em abundância no substrato de fundo e margem neste estudo. A presença deste grupo é comum no silte, mas também é encontrada em macrófitas (de Souza Franco \& Takeda, 2018), corroborando os resultados. Pantala, que foi o terceiro gênero com maior abundância numérica nos tanques, é considerado o mais comum 
nesses ambientes e o principal predador de peixes em seus estágios iniciais de vida, é um gênero periférico e pode colonizar todos os tipos de ecossistemas aquáticos de água doce (Lacerda et al., 2011; Oliveira et al., 2013). Os demais gêneros de Libellulidae encontrados também são comuns em ambientes de piscicultura e possuem maior relação com o substrato (de Marco Junior et al., 1999; Tomazelli et al., 2011).

Em relação às outras duas famílias presentes nos tanques como Aeshnidae (Anax) e Coenagrionidae (Achantagrion e Ischnura) o número de imaturos coletados foi baixo e só estiveram associados aos tanques onde havia larvas, pós-larvas e alevinos de tilápia e maior porcentagem de S. auriculata. Em trabalho comparativo com lagoas agrícolas no sul do Brasil e ambientes lóticos, Pires et al. (2013), observaram uma alta frequência de Achantagrion em fazendas agrícolas e também a presença de Ischnura e Anax. O gênero Anax pode ser considerado comum em tanques de piscicultura, suas larvas atingem grande porte e é um predador eficiente (Santos et al., 1988).

Tabela 2. Relação entre os gêneros de Odonata e os microhabitats (macrófitas e substrato) em que foram encontrados nas amostras em cinco tanques de piscicultura em Alfenas-MG.

\begin{tabular}{|c|c|c|}
\hline Larvas & Macrófita & Substrato \\
\hline \multicolumn{3}{|l|}{ AESHNIDAE } \\
\hline Anax Leach in Brewster, 1815 & 2 & 0 \\
\hline \multicolumn{3}{|l|}{ COENAGRIONIDAE } \\
\hline Achantagrion Selys, 1876 & 21 & 0 \\
\hline Ischnura Charpentier, 1840 & 11 & 0 \\
\hline \multicolumn{3}{|l|}{ LIBELLUIDAE } \\
\hline Brachymesia Kirby, 1889 & 30 & 9 \\
\hline Erythemis Hagen, 1861 & 32 & 113 \\
\hline Erythrodiplax Brauer, 1868 & 7 & 22 \\
\hline Miathyria Kirby, 1889 & 154 & 86 \\
\hline Micrathyria Kirby, 1889 & 22 & 0 \\
\hline Orthemis Hagen, 1861 & 5 & 40 \\
\hline Pantala Hagen, 1861 & 37 & 46 \\
\hline Perithemis Hagen, 1861 & 25 & 10 \\
\hline Tauriphila Kirby, 1889 & 19 & 1 \\
\hline
\end{tabular}

Fonte: Dados da pesquisa.

$\mathrm{Na}$ Análise de Correspondência Simples (Figura 2), os dois primeiros eixos descrevem 84,04\% dos dados, sendo que o primeiro explica $73,90 \%$ e o segundo $14,14 \%$. Dois grupos foram formados. O eixo 1 foi associado aos gêneros Erythrodiplax, Perithemis, Brachymesia, Tauriphila, Achantagrion, Ischnura e Anax. O Eixo 2 foi associado com Miathyria, Erythemis, Pantala, Orthemis e Micrathyria. Os tanques foram segregados, Miathyria, Erythemis, Pantala, Erythrodiplax e Brachymesia foram abordados em todos os tanques, Micrathyria e Orthemis com tanques 1 e 2, e Tauriphila, Achantagrion, Ischnura e Anax com o tanque 3, 4 e 5.

A Análise de Correspondência Simples foi capaz de associar claramente os gêneros aos tanques com a formação de grupos distintos. 
Figura 2. Análise de Correspondência Simples entre os tanques escavados de piscicultura com os gêneros de Odonata encontrados na mesorregião de Alfenas/MG.

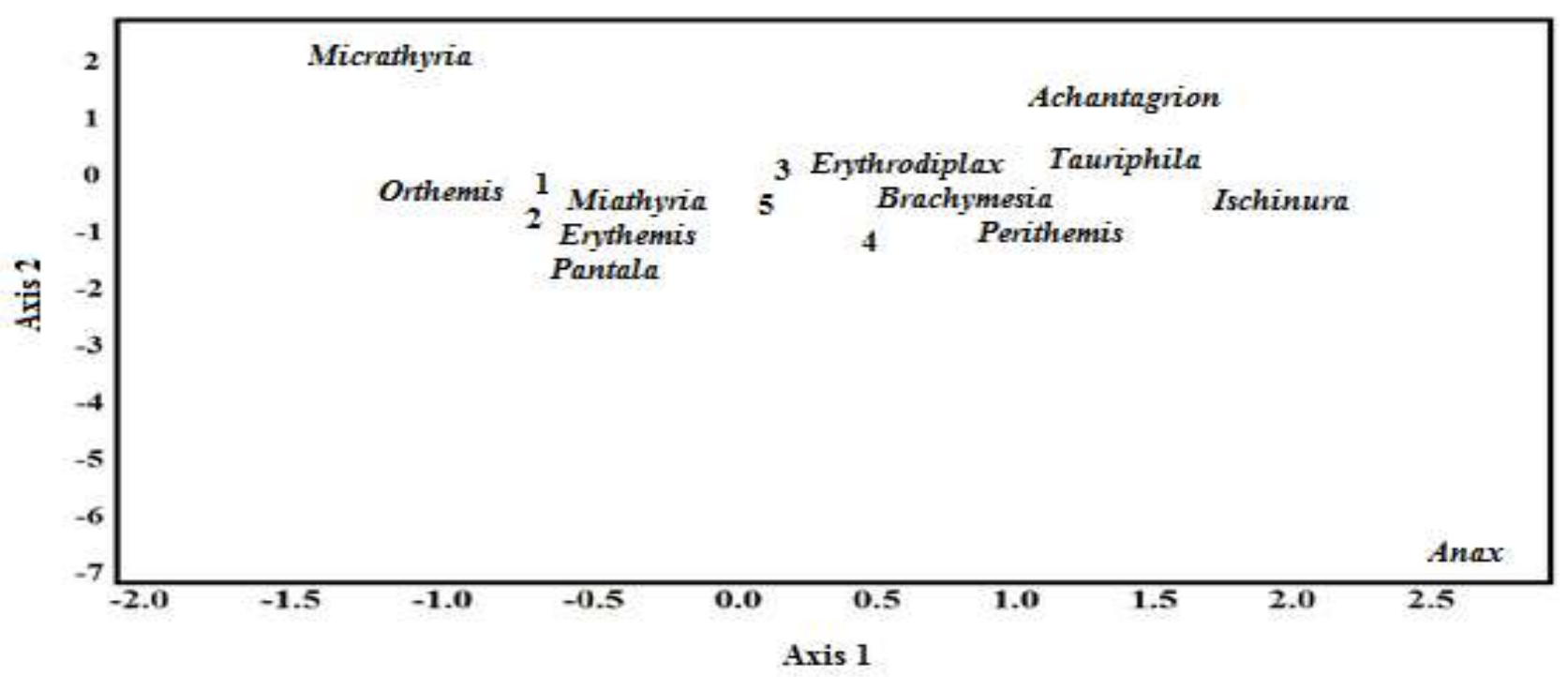

Fonte: Análise dos dados da pesquisa.

Em relação às variáveis físico-químicas (Tabela 3) dos tanques de piscicultura, houve semelhanças quanto às taxas médias de oxigênio dissolvido entre eles, assim como a temperatura e o pH. Porém, a amônia tóxica apresentou valores baixos nos tanques 1 e 2 e extremamente baixos no tanque 5. Havia nitrito apenas no tanque 2.

O oxigênio dissolvido no tanque 2 foi o único que distanciou a taxa média entre os demais e apresentou menor frequência de gêneros, porém, quando comparado ao tanque 1, que também apresentou baixa frequência de gêneros, o valor médio de oxigênio dissolvido foi superior e, portanto, não é possível afirmar que esta variável foi responsável pela baixa abundância de gêneros nesses dois tanques, enquanto nos tanques 3, 4 e 5, os valores de oxigênio dissolvido foram semelhantes e podem ter contribuído, além dos fatores mencionados acima, à abundância numérica dos gêneros.

Os valores de oxigênio dissolvido podem influenciar diretamente na composição de Odonata, uma vez que Anisoptera possui um sistema respiratório específico para retirar oxigênio da água, que pode ser simples em Cordulegastridae, papilar em Gomphidae ou duplo em Libellulidae (Corbet, 1999). Em Zygoptera, a respiração ocorre por meio de movimentos retais que impulsionam a água para um epitélio ricamente irrigado, alguns Zygoptera como Coenagrionidae podem compensar a baixa oxigenação aumentando a frequência e amplitude dos movimentos retais (Corbet, 1999).

A maior toxicidade de amônia nos tanques 1 e 2 pode estar relacionada à ausência de macrófitas, visto que possuem potencial de retenção devido às suas raízes e remoção de poluentes pelo exterior, o mesmo ocorre com o nitrito, porém seus valores foram baixos (Hussar \& Bastos, 2008). Segundo Proença \& Bittencourt (1994), é recomendado que o teor de nitrito em ambientes de piscicultura não ultrapasse $0,15 \mathrm{mg} / \mathrm{L}$ e amônia até $0,05 \mathrm{mg} / 1$.

Os valores de $\mathrm{pH}$ entre os tanques variaram pouco, este é um parâmetro importante no ecossistema aquático, pois é capaz de determinar a dissolução, precipitação, oxidação e redução de várias substâncias no metabolismo dos organismos aquáticos (Bourg \& Loch, 1995; Gill, 1996; Weiner, 2000).

Tabela 3. Médias das variáveis físicas e químicas dos tanques de piscicultura analisadas entre os meses de out/18 a mar/19 na Universidade José do Rosário Vellano, mesorregião do Sul de Minas Gerais, Brasil. 


\begin{tabular}{c|c|c|c|c|c}
\hline Tanques & $\begin{array}{c}\text { Oxigênio } \\
\text { dissolvido }(\mathbf{m g} / \mathbf{L})\end{array}$ & $\begin{array}{c}\text { Temperatura } \\
\left({ }^{\circ} \mathbf{C}\right)\end{array}$ & $\begin{array}{c}\text { Amônia } \\
\text { tóxica }\end{array}$ & Nitrito & $\mathbf{p H}$ \\
\hline Tanque 1 & 6 & 23.4 & 0.02 & 0 & 6.6 \\
Tanque 2 & 5.6 & 23.9 & 0.03 & 0.04 & 6.9 \\
Tanque 3 & 6.3 & 23.7 & 0 & 0 & 6.4 \\
Tanque 4 & 6 & 24 & 0 & 0 & 6.9 \\
Tanque 5 & 6 & 24.2 & 0.08 & 0 & 6.6 \\
\hline
\end{tabular}

Fonte: Dados da pesquisa.

$\mathrm{Na}$ Análise de Componentes Principais (Figura 3), os dois eixos explicam 99,95\% dos dados, com o eixo 1 representando 65,75\% e o eixo 2 34,20\%. O componente 1 foi associado à amônia tóxica com os tanques 3 e 4 e nitrito com o tanque 1 com o tanque 5 isolado. O componente 2 centralizou o oxigênio dissolvido, temperatura e pH, enquanto o tanque 2 foi isolado.

A Análise de Componentes Principais (ACP) correlacionou de forma eficiente às médias das variáveis físicas e químicas com os tanques de piscicultura. Para as variáveis físicas e químicas, todos os dados, exceto temperatura, estão dentro do estabelecido pelo CONAMA nº 357/05 para qualidade da água em ambientes lênticos.

Figura 3. Análise dos Componentes Principais entre as variáveis físicas e químicas e os tanques de piscicultura no Sul de Minas Gerais, onde houveram coletas de larvas de Odonata entre os meses de out/18 a mar/19.

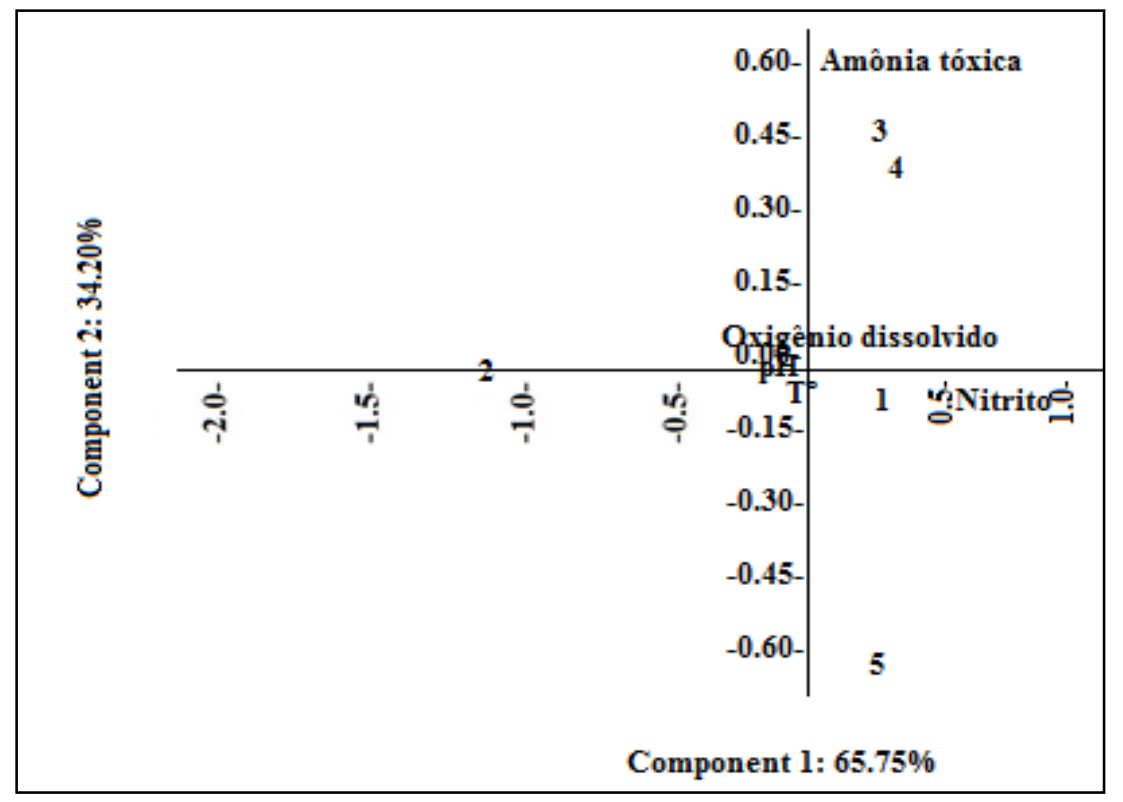

Fonte: Análise dos dados da pesquisa.

Quanto às variáveis ambientais, os dados meteorológicos (Tabela 4) indicaram baixa variação de temperatura entre os dias em que ocorreram as coletas, assim como precipitação. Para a umidade relativa do ar, os índices ficaram apenas abaixo de $70 \%$ no terceiro e no quarto dia de coleta. Na velocidade do vento ocorreram taxas maiores no terceiro dia de coleta e taxas menores no sexto dia de coleta.

A temperatura do ar pode ter influenciado os índices não tão altos de temperatura da água nos tanques entre os dias de coleta, desde a verificação dos valores de temperatura do ar pela estação COOXUPÉ, pois, nos dias anteriores e posteriores ao período de coleta, houve temperaturas mais altas. A tilápia do Nilo (Oreochromis niloticus) pode crescer em temperaturas mais 
baixas, mas se desenvolve melhor em temperaturas entre $26^{\circ} \mathrm{C}$ a $28{ }^{\circ} \mathrm{C}$, já o Curimba precisa de temperatura mais altas, para Odonata é necessária uma temperatura acima de $15{ }^{\circ} \mathrm{C}$ para que as larvas se desenvolvam, porém, algumas espécies chegam abaixo deste valor (Krishnaraj et al., 1995; Justi et al., 2005; Mendonça et al., 2018; Silva et al., 2016).

Em relação aos índices de chuva e velocidade do vento nos dias de coleta, houve apenas chuva no momento da coleta em outubro de 2018, porém nos 6 meses de trabalho, a quantidade $(\mathrm{mm})$ de chuva e a velocidade do vento na mesorregião de Alfenas-MG variaram conforme dados da COOXUPÉ e essa variação pôde influenciar na diversidade de Odonata, bem como na alteração dos parâmetros físico-químicos (Kalkman et al., 2008; Fulan et al., 2007; Carvalho et al., 2000).

Tabela 4. Dados meteorológicos de Alfenas-MG dos dias de coletas de Odonata em tanques de piscicultura da Universidade José do Rosário Vellano.

\begin{tabular}{c|c|c|c|c}
\hline Dias de coleta & $\begin{array}{c}\text { Temperatura do ar } \\
\left({ }^{\circ} \mathbf{C}\right)\end{array}$ & Precipitação $(\mathbf{m m})$ & $\begin{array}{c}\text { Humidade relativa } \\
\mathbf{d o} \text { ar }(\boldsymbol{\%})\end{array}$ & $\begin{array}{c}\text { Vento máximo } \\
(\mathbf{k m} / \mathbf{h})\end{array}$ \\
\hline $18 / 10 / 2018$ & 28.3 & 10.4 & 79 & 25.7 \\
\hline $23 / 11 / 2018$ & 28.1 & 0.2 & 71 & 25.7 \\
\hline $08 / 12 / 2018$ & 27.9 & 0.4 & 62 & 27.4 \\
\hline $09 / 01 / 2019$ & 31.1 & 0 & 60 & 22.5 \\
\hline $07 / 02 / 2019$ & 28.6 & 0.2 & 79 & 25.7 \\
\hline $13 / 03 / 2019$ & 27.9 & 2 & 70 & 16.1 \\
\hline
\end{tabular}

Fonte: Estação Meteorológica COOXUPÉ (2020).

\section{Considerações Finais}

Dessa forma, pode-se concluir que existe uma dominância de Libelluidae nesses ambientes de piscicultura, tanto em macrófitas quanto em substratos, mas os gêneros mais associados ao substrato devem ser levados em consideração, uma vez que em ambientes de piscicultura há controle rígido das macrófitas. Miathyria mostrou-se generalista em relação aos dois microhabitats, enquanto Erythemis estava mais associada ao substrato. Também foi possível observar que às variáveis ambientais podem ter influenciado as variáveis físicas e químicas, principalmente na temperatura da água. Vale ressaltar que mais estudos sobre o levantamento de Odonata em tanques de peixes e para a aquicultura em geral são necessários, tanto a nível de gênero e espécie, quanto estudos que compreendam os níveis de predação de Odonata em peixes e em outros organismos aquáticos de produção, a fim de resolver esta questão de uma maneira sustentável.

\section{Agradecimentos}

À CAPES (Coordenação de Aperfeiçoamento de Pessoal de Nível Superior) pelo financiamento do projeto (processo nº 88882.365356 / 2019-01), à Universidade José do Rosário Vellano pelo apoio e infraestrutura.

\section{Referências}

Américo, J. H. P., Torres, N. H., Machado, A. A., \& Carvalho, S. L. (2013). Piscicultura em tanques-rede: impactos e consequências na qualidade da água. Revista Científica ANAP Brasil, 6 (7).

Andrade, D. R., \& Yasui, G. S. (2003). Manejo da reprodução natural e artificial e sua importância na produção de peixes no Brasil. Revista Brasileira de Reprodução Animal, 27(2), 166-172.

Borror, D. J., Triplehorn, C. A., \& Johnson, N. F. (1989). An introduction to the study of insects (No. Ed. 6). Saunders college publishing.

Bourg, A. C. M., \& Loch, J. G. (1995). Mobilization of heavy metals as affected by $\mathrm{pH}$ and redox conditions. In Biogeodynamics of pollutants in soils and sediments (pp. 87-102). Springer, Berlin, Heidelberg. 
Brandão, C. J., Botelho, M. J. C., \& Sato, M. I. Z. (2018). Guia nacional de coleta e preservação de amostras: água, sedimento, comunidades aquáticas e efluentes líquidos.

Carvalho, A. L., \& Pessacq, P. (2018). Superfamily Aeshnoidea. In: Hamada, N., Thorp, J. H., \& Rogers, D. C. (eds.) Thorp and Covich's Freshwater Invertebrates: Keys to Neotropical Hexapoda. (4a ed.), Lawrence, Academic Press, 3, 367-376.

Carvalho, A. R., Schlittler, F. H. M., \& Tornisielo, V. L. (2000). Influence of cattle ranching and agricultural activities on physical chemical parameters of water. Química Nova, 23(5), 618-622.

Conama- Conselho Nacional do Meio Ambiente. (2005). Resolução ${ }^{\circ}$ 357. http://www.2mma.gov.br/por/conama/legiabre.cfm?codlegi=459

Cooxupé - Cooperativa Regional de Cafeicultores de Guaxupé. (2020). Estações Meteorológicas-dados históricos. http://sismet.cooxupe.com.br

Corbet, P. S. (1999). Dragonflies: behaviour and ecology of Odonata. Harley books.

Costa, J. M., Santos T. C., \& Oldrini, B. B. Odonata. (2012). In: Rafael, J. A., Melo, G. A. R., Carvalho, C. J. B., Casari, S. A., \& Constantino, R. Insetos do Brasil: Diversidade e Taxonomia. Holos Editora. 1, 246- 256.

Da Silva, L. V. F., Fernandes, M. N., \& Baldisserotto, B. (2009). Incubação e desenvolvimento de peixes aplicados à piscicultura: necessidades e cuidados. Manejo e sanidade de peixes em cultivo, 64.

De Faria, C. F. A., Pontes, C. S., Ribeiro, K., \& de Faria, P. M. C. (2019). Caracterização do mercado de aquicultura ornamental e aquariofilia no rio grande do norte. Revista Gestão \& Sustentabilidade Ambiental, 8(1), 203-215.

De Marco Jr, P., Latini, A. O., \& Reis, A. P. (1999). Environmental determination of dragonfly assemblage in aquaculture ponds. Aquaculture Research, 30(5), 357-364.

De Marco Jr, P., \& Resende, D. C. (2002). Activity patterns and thermoregulation in a tropical dragonfly assemblage. Odonatologica, 31(2), 129-138.

De Souza Franco, G. M., \& Takeda, A. M. (2002). Variação espacial e temporal de larvas de Odonata associadas com macrófitas aquáticas em duas lagoas da planície de inundação do alto rio Paraná, Brasil. Acta Scientiarum. Biological Sciences, 24, 345-351.

Fao., Fida., Unicef., Pms., \& Oms. (2018). El estado de la seguridad alimentaria y la nutrición en el mundo. Fomentando la resiliencia climática en aras de la seguridad alimentaria la nutrición. http://www.fao.org/3/19553ES/i9553es.pdf

Fonseca, A. R., Sanches, N. M., da Fonseca, M. C., Quintilhiano, D. M., \& da Silva, E. S. (2004). Levantamento de espécies de Odonata associadas à tanques de piscicultura e efeito de Bacillus thuringiensis var. israelensis sobre ninfas de Pantala flavescens (Fabricius, 1798. Acta Scientiarum. Biological Sciences, 26(1), 25-29.

Fulan, J. Â., \& Henry, R. (2007). Distribuição temporal de imaturos de Odonata (Insecta) associados a Eichhornia azurea (Kunth) na lagoa do Camargo, rio Paranapanema, São Paulo. Revista Brasileira de Entomologia, 51, 224-227.

Gill, R. (1996). Chemical fundamentals of geology (No. $550.4 \mathrm{GIL})$.

Hammer, Ø., Harper, D. A., \& Ryan, P. D. (2001). PAST: Paleontological statistics software package for education and data analysis. Palaeontologia electronica, 4(1), 9 .

Hussar, G. J., \& Bastos, M. C. (2008). Tratamento de efluente de piscicultura com macrófitas aquáticas flutuantes. Engenharia Ambiental, 5(3), $274-285$.

Igarashi, M. A. (2018). Aspectos tecnológicos e perspectivas de desenvolvimento do cultivo de tilápia no Brasil. Arquivos de Ciências Veterinárias e Zoologia da UNIPAR, 21(3).

Justi, K. C., das Graças Padre, R., Hayashi, C., Soares, C. M., Visentainer, J. V., de Souza, N. E., \& Matsushita, M. (2005). Efeito da temperatura da água sobre desempenho e perfil de ácidos graxos de tilápia do Nilo (Oreochromis niloticus). Acta Scientiarum. Animal Sciences, 27(4), 529-534.

Kalkman, V. J., Clausnitzer, V., Dijkstra, K. D. B., Orr, A. G., Paulson, D. R.; \& Van Tol, J. Global diversity of dragonflies (Odonata) in freshwater. In: Bailan, E.V.; Lévêque, C.; Sergers, K.; Martens, K. (eds.) Freshwater animal diversity assessment, Springer, Dordrecht, 2008. 198, 351-363.

Kirchner, R. M., Chaves, M. A., Silinske, J., Essi, L., Scherer, M. E., \& Durigon, E. G. (2016). Análise da produção e comercialização do pescado no Brasil.RevistaAgro@ mbiente On-line, 10(2),168-177.

Krishnaraj, R., \& Pritchard, G. (1995). The influence of larval size, temperature, and components of the functional response to prey density on growth rates of the dragonflies Lestes disjunctus and Coenagrion resolutum (Insecta: Odonata). Canadian journal of zoology, 73(9), 1672-1680.

Kubitza, F. (2003). Qualidade da água no cultivo de peixes e camarões. F. Kubitza.

Kubitza, F. (2004). Reprodução, larvicultura e produção de alevinos de peixes nativos. Jundiaí: Acqua e imagem.

Kubitza, F. (2011). Tilápia: Tecnologia e planejamento na produção comercial. (2a ed.), Acqua Supre Com. Suprim. Aquicultura.

Lacerda, C. H. F., Hayashi, C., Galdioli, E. M., \& Fernandes, C. E. B. (2011). Predation of Piaractus mesopotamicus and Oreochromis niloticus larvae by Pantala flavescens with different length classes. Acta Scientiarum. Biological Sciences, 33(4), 377-382.

Lopes, J. P., Siqueira, K. B., Oliveira, L. C. F., \& Brito, J. T. M. (2007). Análise da predação de Notonecta sp. Sobre Juvenis De Tilápia, Variedade "QAAT 1". Revista Brasileira de Engenharia de Pesca, 2(2), 46-51. 
Lozano, F., Muzón, J., Anjos-Santos, D., \& Pessacq, P (2018). Superfamily Coenagrionoidea. In: Hamada, N., Thorp, J. H., \& Rogers, D. C. (eds.). Thorp and Covich's Freshwater Invertebrates: Keys to Neotropical Hexapoda. (4a ed.), Lawrence, Academic Press. 3. $475-494$.

Lui, T. A., de Freitas, J. M. A., Bittencourt, F., Dallagnol, J. M., Neu, D. H., \& Boscolo, W. R. (2018). Índice de acidez do óleo de peixe na nutrição de alevinos de tilápia. Agrarian, 11(40), 174-180.

Mello, S. C. R. P., de Oliveira, E. D. C. P., \& de Seixas Filho, J. T. (2017). Aspectos da aquicultura e sua importância na produção de alimentos de alto valor biológico. Semioses, 11(2), 28-34.

Mendonça, F. Z., Bernardy, J. V., Oliveira, C. E. K., Oliveira, P. B. G., \& De Marco, P. (2018). Temperature effect on the development of tropical dragonfly eggs. Neotropical entomology, 47(4), 484-491.

Meurer, F., Hayashi, C., \& Boscolo, W. R. (2003). Influência do processamento da ração no desempenho e sobrevivência da tilápia do Nilo durante a reversão sexual. Revista Brasileira de Zootecnia, 32, 262-267.

Neiss, U. G., Fleck, G., Pessacq, P., \& Tennessen, K. J. (2018). Odonata: Superfamily Libelluloidea. In: In: Hamada, N.; Thorp, J. H.; Rogers, D. C. (eds.) Thorp and Covich's Freshwater Invertebrates: Keys to Neotropical Hexapoda. (4a ed.), Lawrence, Academic Press. 3, $399-447$.

Olaya, M. (2019). Odonatos en Latinoamérica: la riqueza de nuestra región. Hetaerina, 1(2), 4-5.

Oliveira, E., Takeuchi, S. S., \& Cerutti, V. E. (2013). Assembleia de Larvas de Odonata (Insecta) em ambientes límnicos do Parque Estadual de Vila Velha, Paraná, Brasil. Estudos de Biologia, 35(85).

Ostrensky, A., \& Boeger, W. A. (1998). Piscicultura: fundamentos e técnicas de manejo.

Paulson, D., \& Schorr, M. (2021). World Odonata List. https:// www.pugetsound.edu/academics/academicresources/slatemuseum/biodivers ityresources/dragonflies/world-odonata-list2

Pinder, L. C. V. The habitats of chironomid larvae (1995). In: Armitage, P. D.; Cranston, P. S.; Pinder, L. C. V. The Chironomidae Biology and Ecology of Non-biting Midges. Dordrecht, Springer. 1, 107-135.

Pires, M. M., Kotzian, C. B., Spies, M. R., \& Neri, D. B. (2013). Diversity of Odonata (Insecta) larvae in streams and farm ponds of a montane region in southern Brazil. Biota Neotropica, 13, 259-267.

Proença, C. E. M., \& Bittencourt, P. R. L. (1994). Manual de piscicultura tropical. IBAMA. DIREN/DEPAQ/DIPEA.

Queiroz, J. C. (2017). Controle químico de ninfas de libélula (Insecta, Odonata) durante a larvicultura do Jundiá (Rhamdia quelen). 62f. Dissertação (Mestrado em Zootecnia) - Universidade Estadual do Oeste do Paraná, 2017.

Ramírez, A. (2010). Capítulo 5: Odonata. Revista de Biología Tropical, 58, 97-136.

Sandoval, J. P., Trombeta, T. D., \& de Mattos, B. O. Manual de criação de peixes em tanques-rede. CODEVASF, 2019.

Santos, N. D., Costa, J. M., \& Pujol-Luz, J. R. (1988). Nota sobre a ocorrência de odonatos em tanques de piscicultura e o problema da predação de alevinos pelas larvas. Acta Limnologica Brasiliensia, 2, 771-780.

Schulter, E. P., \& Vieira Filho, J. E. R. (2017). Evolução da piscicultura no brasil: diagnóstico e desenvolvimento da cadeia produtiva de tilápia (No. 2328). Texto para Discussão.

Sebrae - Serviço Brasileiro de Apoio às Micro e Pequenas Empresas. (2015). Aquicultura no Brasil série estudos mercadológicos. http://www.bibliotecas.sebrae.com.br/chronus/ARQUIVOS_CHRONUS/bds/bds.nsf/4b14e85d5844cc99cb3 2040a4980779f/\$File/5403.pdf.

Senar-197. (2017). Piscicultura: reprodução, larvicultura e alevinagem de tilápias. Coleção SENAR.

Senar-198. (2017). Piscicultura: reprodução, larvicultura e alevinagem de peixes nativos. Coleção SENAR.

Silva, T. S. C., Inoue, L. A. K. A., \& Borghesi, R. (2016). Inverno: período crítico para piscicultura. Texto para discussão. https://www.embrapa.br/busca-de noticias//noticia/13478025/artigo---inverno-periodo-critico-para-piscicultura

Siqueira, T. V. (2018). Aquicultura: a nova fronteira para produção de alimentos de forma sustentável. R. BNDS, 25(29), 119-170.

Souza, L. O. I., Costa, J. M., \& Oldrini, B. B. (2007). Odonata. Guia on-line: Identificação de larvas de Insetos Aquáticos do Estado de São Paulo.

Tachibana, L., Castagnolli, N., Pezzato, L. E., Barros, M. M., de Barros Valle, J., \& Siqueira, M. R. (2004). Desempenho de diferentes linhagens de tilápia do Nilo (Oreochromis niloticus) na fase de reversão sexual. Acta Scientiarum. Animal Sciences, 26(3), 305-311.

Tachibana, L., Leonardo, A. F. G., Corrêa, C. F., \& Saes, L. A. (2018). Densidade de estocagem de pós-larvas de tilápia-do-Nilo (Oreochromis niloticus) durante a fase de reversão sexual. Boletim do Instituto de Pesca, 34(4), 483-488.

Tave, D., Rezk, M., \& Smitherman, R. O. (1990). Effect of body colour of Oreochromis mossambicus (Peters) on predation by dragonfly nymphs. Aquaculture Research, 21(2), 157-162.

Trueman, J. W. H., \& Rowe, R. J. (2009). Odonata. Dragonflies and damselflies. The Tree Life Web Project. http://tolweb.org/Odonata/8266/2009.10.16

Tomazelli Júnior, O., Franco, G. M. S., Casaca, J. M., Munarini, A. C., \& Magro, J. D. (2011). Effect of the Melia azedarach L. on the predation of common carp fingerlings (Cyprinus carpio) by larvae of Neuraeschna (Odonata: Aeshnidae). Brazilian Journal of Aquatic Science \& Technology, 15(1), 19-25. 
Research, Society and Development, v. 10, n. 11, e363101119846, 2021

(CC BY 4.0) | ISSN 2525-3409 | DOI: http://dx.doi.org/10.33448/rsd-v10i11.19846

Veloso, H. P., Rangel-Filho, A. L. R., \& Lima, J. C. A. (1991). Classificação da vegetação brasileira, adaptada a um sistema universal. Ibge.

Weiner, R., Taylor, L., Ekborg, N., \& Whitehead, L. (2000). Degradosomes: potential importance in the ocean's carbon cycle and in aquaculture and algal culture. Recent advances in marine science and technology, 259-268.

Zaniboni Filho, E., \& Weingartner, M. (2007). Técnicas de indução da reprodução de peixes migradores. Revista Brasileira de Reprodução Animal, 31(3), 367-373. 